\title{
MOTIVACIONES DE LOS JÓVENES SEVILLANOS PARA ABANDONAR LA PRÁCTICA DE ACTIVIDAD FÍSICO-DEPORTIVA
}

\section{MOTIVATIONS FOR SEVILLIAN YOUNG PEOPLE TO ABANDON PHYSICAL ACTIVITY PRACTICE}

Gavala González, Juan

Doctor por la Universidad Pablo de Olavide. Universidad Pablo de Olavide (jgavgon@upo.es)

\author{
Código UNESCO: 5802.99 \\ Clasificación Consejo de Europa: 17. Actividad Física y Salud.
}

Recibido el 6 de junio de 2012

Aceptado el 29 de agosto de 2012

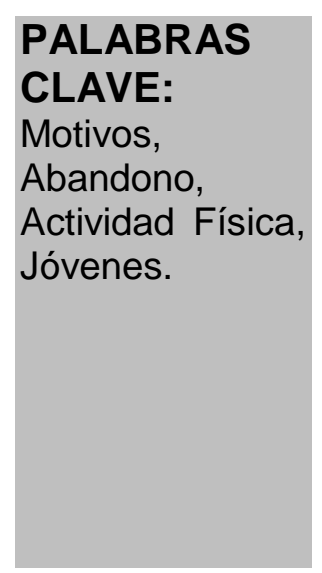

KEY WORDS:

Reasons,

Abandonment,

Physical activity,

Young people.

\begin{abstract}
RESUMEN
Éste estudio analiza las razones que llevan a los jóvenes sevillanos (6-18 años) a abandonar la práctica físico-deportiva. Para ello hemos realizado un muestreo polietápico, con selección de las unidades primarias (centros escolares) por distritos municipales y tipología de centro, según un muestreo aleatorio simple, aplicando afijación proporcional. Las unidades secundarias (los individuos) se han seleccionado mediante un censo aplicando cuotas por género e intervalos de edad. Tras la aplicación de diversas técnicas estadísticas, la muestra debía componerse de 1.074 individuos asumiendo un margen de error del $+2.97 \%$ para un nivel de confianza del 95\%. El método de recogida de información consistió en cuestionarios/entrevista personal. Los resultados más relevantes, al igual que las diversas investigaciones consultadas, fueron que la mayoría de los jóvenes sevillanos abandonan debido a la desmotivación y a la falta de tiempo, por este orden, mientras que en la numerosa bibliografía consultada, estos motivos invierten su orden.
\end{abstract}

\section{ABSTRACT}

In this article, we study the reasons that lead young people of Seville (6 to 18 years) to abandon the practice of physical and sports activities. We therefore performed a multistage sampling, with selection of primary units (schools) for municipal districts and type of center, according to a simple random sampling, using proportional representation. Secondary units (individuals) are selected by a survey using quotas by gender and age ranges. After applying statistical techniques, we conclude that the sample was composed of 1,074 individuals that we guarantee a margin of error of $\pm 2.97 \%$ for a confidence level of $95 \%$. The method of data collection consisted of questionnaires and personal interview. And the most relevant results, as well as the various investigations consulted, was that most young people stop practicing Seville due to demotivation and lack of time, in that order, while in the large bibliography, these two reasons reverse their order. 


\section{INTRODUCCIÓN}

El abandono deportivo se produce por una relación entre varios factores ${ }^{(1)}$ aunque, normalmente el abandono es debido a la percepción que tiene el sujeto sobre sus circunstancias. De este modo, la intención participar en una actividad físico-deportiva junto con la percepción de facilidad o dificultad a la hora de realizarla son los mejores predictores para conocer si el sujeto tendrá adherencia a dicha actividad o la

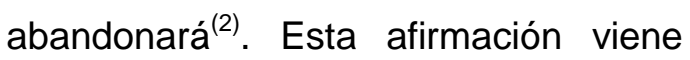
respaldada por varios trabajos que afirman que aquellos sujetos que perciben más dificultades son los que en mayor proporción abandonaran dicha actividad en el momento en el que aparezca dicha percepción de las dificultades $^{(3,4,1)}$.

Otro dato interesante es que el abandono deportivo corresponde frecuentemente a épocas en las que se producen cambios en la vida de las personas (cambio de estudios obligatorios a opcionales, la incorporación al mundo laboral, etc.) ${ }^{(5)}$. Y la adolescencia, entre los 12 y los 18 años, es una etapa de cambios fisiológicos, psíquicos y sociales y donde se produce un incremento del abandono de la práctica físicodeportiva en ambos sexos ${ }^{(6,7,8,9,1)}$.

Rev. lb. CL. Act.Fis. Dep.
En relación a las razones que llevan a las personas a abandonar este tipo de actividades, numerosos estudios han encontrado que la principal razón es la falta de tiempo, seguida de la pérdida de interés total por la actividad físicodeportiva (abandono total) o por una en concreto(abandono relativo) 2,5,10,11,12,13,14 Lo que hace que la persona pase a realizar otra nueva actividad porque la que abandona no satisfacía sus necesidades $^{(15,16,17,18,19)}$.

Existen otros estudios en los que la falta de tiempo viene acompañada por otro motivo como pueden ser la falta de instalaciones ${ }^{(20,21)}$, el miedo a las lesiones $^{(22)}$ o el tener muchos deberes $y$ tener que estudiar mucho ${ }^{(2)}$.

\section{METODOLOGÍA}

En la literatura existente en temas de muestreo se considera que esta reducción no influye en el estudio de los hábitos deportivos generales de la población joven del municipio. Con todo ello, la población objeto de estudio asciende a 85.744 individuos, pudiéndose observar, en la base de datos facilitada por la Unidad de Sistemas de Información de la Consejería de Educación de la Junta de Andalucía, su distribución según distritos, grupos de edad y titularidad del centro de estudios, así como su 
distribución según distrito, género y titularidad.

Para analizar las posibles características diferenciales que se pueden presentar en los distintos distritos en los que se divide la capital sevillana y teniendo en cuenta la estructura de la población investigada según la tipología de centro escolar, se ha optado por realizar un muestreo polietápico.

En una primera fase se ha realizado un muestreo estratificado con afijación proporcional seleccionando 22 estratos. Cada estrato está formado por colegios, que son las unidades muestrales primarias, resultado del cruce entre cada uno de los 11 distritos existentes en Sevilla capital y el tipo de colegio, público o privado-concertado al que asisten los individuos objeto de estudio. Además se ha aplicado para la obtención de la información cuotas por género y edad.

La decisión de estratificar la población ha sido fundamentada en la posible diferencia que existe en las necesidades de la población que reside en los distintos distritos y las características singulares que se pueden dar por pertenecer a un tipo $u$ otro de colegio por parte de la población investigada. A su vez, ha influido el estudio del entorno urbano de cada distrito y los servicios deportivos y de ocio, disponibles y ofertados, en cada zona geográfica.

Dentro de cada estrato se ha optado por un muestreo aleatorio simple cuyas unidades investigadas han sido los colegios. Para ello se ha utilizado como marco el Censo de Centros Escolares, el cual cuenta con información suplementaria que posibilitará el estudio y la ampliación de la información obtenida a través del trabajo de campo.

Para seleccionar los colegios que finalmente han pertenecido a la muestra se ha considerado la fórmula del tamaño óptimo de muestra para una proporción en el muestreo aleatorio simple.

Como fase previa a la obtención de la información se ha realizado un pretest como herramienta de detección de anomalías e indeterminaciones que pudiese tener el cuestionario utilizado. Finalmente, debido a la puesta en marcha del trabajo de campo, el tamaño efectivo que se obtiene en la investigación es de 1.074 jóvenes.

En una última etapa muestral se ha realizado un censo dentro de cada colegio seleccionado investigado, mediante entrevista personal, a los jóvenes con edades comprendidas entre 6 y 18 años pero aplicando cuotas de edad y género para obtener finalmente una muestra representativa. 
Para ello se han tenido en cuenta las edades según la etapa de estudio y el género, alcanzando de esta forma un equilibrio muy positivo entre los resultados deseables y las restricciones inevitables de tiempo y coste.

\section{OBJETIVOS}

- Conocer los motivos por los que los jóvenes sevillanos abandonan la actividad físicodeportiva.

- Establecer diferencias entre los motivos mencionados en función de diferentes variables (sexo, edad, etapa educativa y centro educativo).

\section{RESULTADOS}

A la hora de preguntar a los jóvenes sevillanos el por qué de su abandono físico-deportivo, hemos encontrado que los motivos más reiterados son la falta de motivación (32.94\%), los problemas con los estudios (26.23\%) y los problemas de salud 0 lesiones (14.79\%). A éstos le siguen otros menos repetidos como son el no tener instalaciones cerca $(7.50 \%)$, problemas con el entorno deportivo (6.90\%), no tener las instalaciones adecuadas $(3.55 \%)$ o problemas con el entorno familiar (3.16\%) y en último lugar aparecen los problemas económicos
(1.38\%) como causa para abandonar la práctica físico-deportiva.

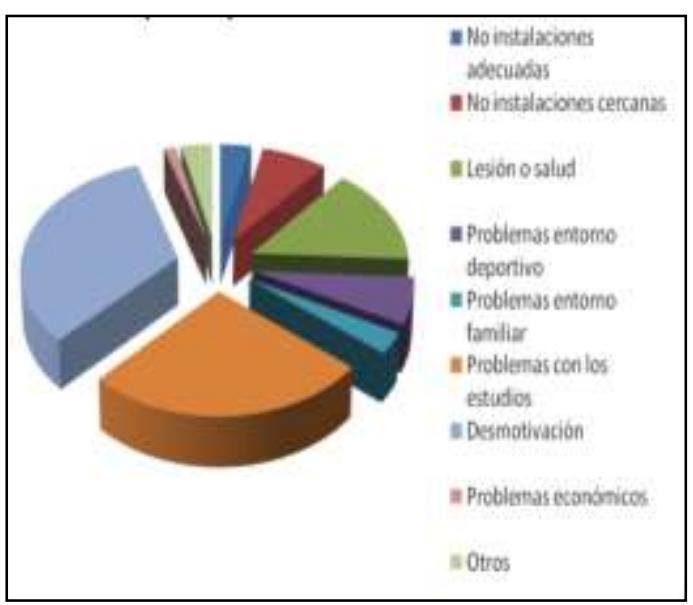

Figura 1. Causas de abandono de la práctica deportiva.

\subsection{Causas de abandono de la} práctica deportiva según género.

Las chicas suelen abandonar su práctica en mayor proporción que los chicos por problemas de salud, desmotivación, mientras que los chicos lo hacen más que ellas por problemas con el entorno deportivo o familiar o por no tener instalaciones adecuadas. En el resto de motivos (problemas con los estudios, económicos, no tener instalaciones cercanas) las diferencias entre uno y otro sexo son ínfimas.

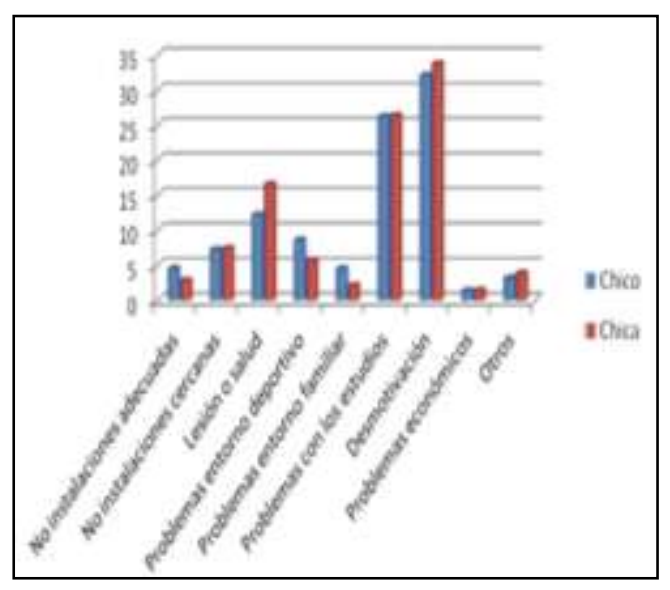

Rev. Ib. CL. Act.Fis. Dep. 
Figura 2. Causas de abandono de la práctica deportiva según género.

\subsection{Causas de abandono de la práctica deportiva según edad.}

En todas las edades los principales motivos de abandono son la desmotivación y los problemas con los estudios, no en vano, son en este orden, las razones más repetidas entre los escolares de 6 a 13 años. Sin embargo, a partir de esta edad, los trece años, invierten su orden de influencia, pasando los problemas con los estudios a ser la causa más repetida, seguida de la otra razón mencionada anteriormente.

Por otro lado, se comprueba que a medida que aumenta la edad la cantidad de los que abandonan por no tener instalaciones cercanas 0 por problemas con los estudios aumenta. Por el contrario disminuyen los que dicen hacerlo por desmotivación, problemas de salud o lesiones, con el entorno familiar, o económicos o por no tener instalaciones adecuadas, en este último caso incluso no se encuentran personas de 17-18 años que digan abandonar por este motivo. Sin embargo, a esta edad aparece una mayor cantidad de personas, en comparación con las anteriores, que dicen no practicar por otros motivos.

Rev. Ib. CL. Act.Fis. Dep.

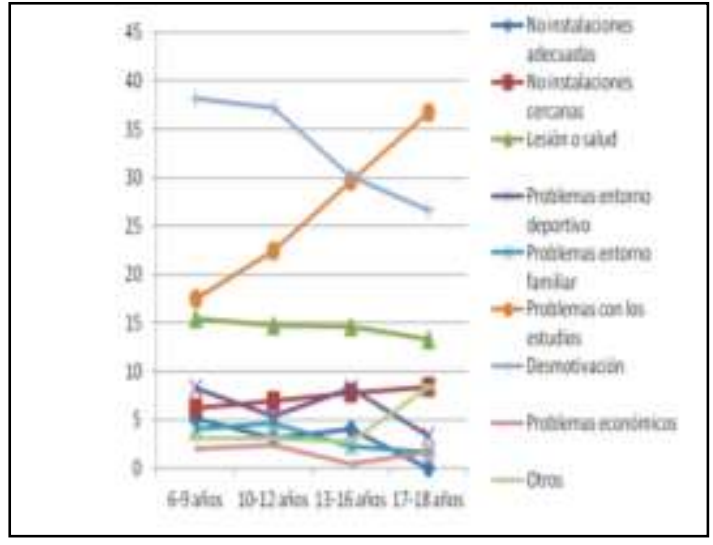

Figura 3. Causas de abandono de la práctica deportiva según edad.

\subsection{Causas de abandono de la práctica deportiva según etapa} educativa.

Dentro de los motivos por los que se abandona según la etapa educativa podemos distinguir tres grupos:

El primero de ellos, formado por los que son más referidos a medida que aumenta el nivel educativo, entre los que están los problemas con los estudios y "otros motivos".

El segundo grupo lo integran las causas que a medida que aumenta el nivel educativo disminuye el número de las personas que dicen referirlas como causante de su abandono, entre las que destacan los motivos de salud 0 lesiones, los problemas con el entorno familiar o los económicos.

Y por último, los que fluctúan en el paso de una etapa a otra, éstos son la desmotivación y los relativos a las instalaciones (no tenerlas cerca o no ser las adecuadas). El primero de ellos 
con un una ligera disminución de la cantidad de alumnos que los nombran entre la primaria y la secundaria para posteriormente aumentar entre esta última y el bachillerato. Por otro lado, los que mencionan los relativos a las instalaciones aumentan en número entre la primaria y la secundaria para posteriormente disminuir e incluso en un caso desaparecer

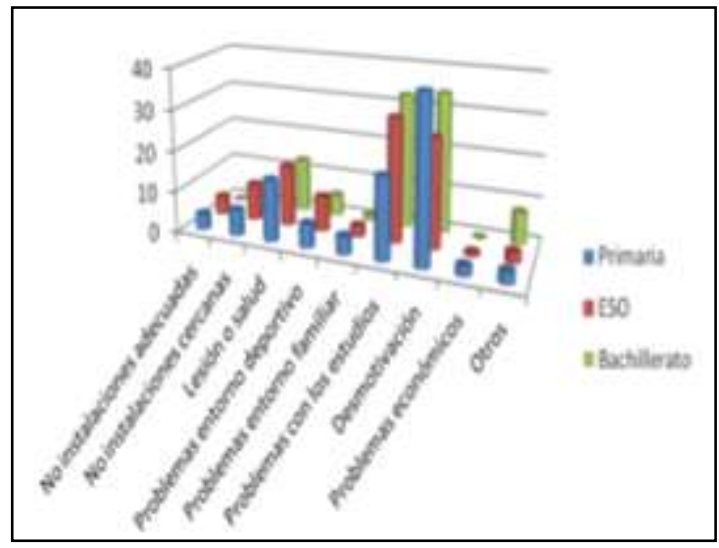

Figura 4. Causas de abandono de la práctica deportiva según etapa educativa.

\subsection{Causas de abandono de la} práctica deportiva según tipo de centro educativo.

Se puede observar cierta similitud entre los alumnados de uno y otro tipo de centro en motivos como no tener instalaciones cercanas, problemas con el entorno familiar, deportivo 0 problemas económicos. Por el contrario existen diferencias en el resto de razones. De este modo, los alumnos de los públicos abandonan en mayor proporción que sus compañeros de los privados-concertados

por desmotivación o problemas de salud o lesiones, mientras que éstos lo hacen en mayor cantidad que aquellos por no tener instalaciones adecuadas y por problemas con los estudios.

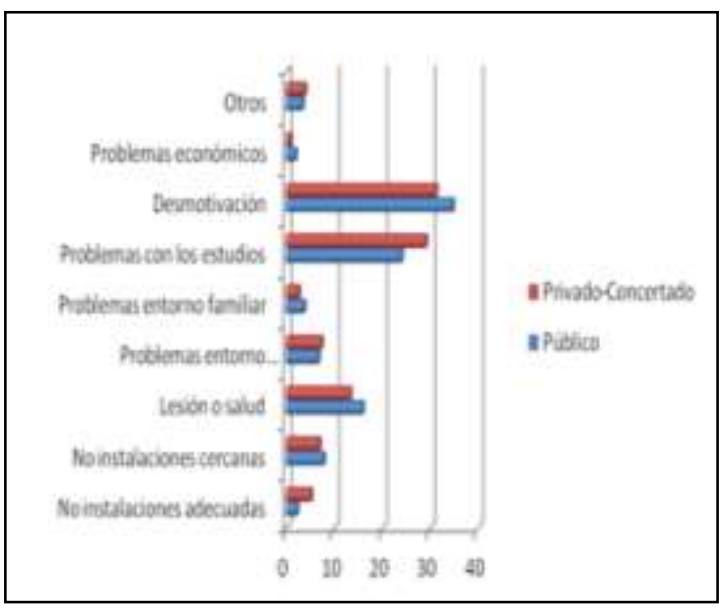

Figura 5. Causas de abandono de la práctica deportiva según tipo de centro educativo.

\section{DISCUSIÓN}

Muchas investigaciones señalan que la razón principal por la que las personas dejan de realizar práctica de actividades físico-deportivas es la falta de tiempo, o la incompatibilidad de dichas actividades con el trabajo o los estudios, siendo el siguiente motivo más mayoritario la pérdida de interés por la actividad físico-deportiva de manera general 0 de manera específica $^{(2,5,10,11,12,13,14)}$.

En cambio, en nuestro estudio estos dos motivos invierten su orden. Los jóvenes sevillanos dejan de practicar en mayor proporción por la 
desmotivación, seguido de la incompatibilidad con los estudios.

En relación a las variables sociodemográficas, podemos encontrar en otras investigaciones en las que el número de mujeres que suele abandonar estas actividades es mayor que el de los varones ${ }^{(1,2,22)}$, debido en muchos casos a que mientras que los chicos perciben en mayor proporción los beneficios de dichas prácticas, las chicas perciben mayoritariamente dificultades para realizarlas ${ }^{(23)}$ asociando la pérdida de feminidad y la discriminación de la mujer en el deporte $^{(6)}$. En nuestra investigación no existen grandes diferencias por las que ambos sexos dejan de practicar, si bien, las chicas registran porcentajes algo superiores a los chicos en los motivos principales.

Por otro lado, cuanto mayor es el nivel educativo existe una mayor tasa de abandono debido a las múltiples actividades que se deben realizar además de las físico-deportivas ${ }^{(5)}$. Nuestro estudio coincide con esta conclusión, pero además va más allá, ya que podemos afirmar que a medida que aumenta el nivel académico aumenta el abandono por incompatibilidad con los estudios, mientras que el otro motivo mayoritario, la desmotivación fluctúa en el paso de las etapas, es decir, disminuye el número de alumnos que dejan este tipo de actividades entre las etapas de primaria y secundaria, sin embargo, en el paso de ésta con el Bachillerato sí aumenta.

Y finalmente, en cuanto a la edad, podemos comprobar que, a medida que aumenta la edad, disminuye la cantidad de personas que refieren la falta de instalaciones como razones para dejar de practicar $^{(2,17)}$. En cambio en nuestro estudio encontramos que, a medida que aumenta la edad, crece el número de los que abandonan por no tener instalaciones cercanas o por falta de tiempo. Asimismo, encontramos otro dato muy interesante, éste es: entre los 6 y los 13 años la mayoría de abandonos se deben a la desmotivación y a la incompatibilidad con el estudio, mientras que entre los 14 y los 18 años, estos motivos invierten su orden, siendo causa de mayor abandono la problemática con los estudios

Además en nuestro estudio hemos podido comprobar que el alumnado de los centros públicos suele abandonar la práctica físico-deportiva por desmotivación o por problemas de salud en mayor cantidad que el 
alumnado de los privados-concertados, que lo suelen hacer por incompatibilidad con los estudios o por no tener instalaciones adecuadas, sin embargo este dato no lo hemos podido contratar con ningún estudio ya que los que han estudiado este tipo de poblaciones no los han relacionado con el tipo de centro.

\section{CONCLUSIONES}

Nuestra investigación coincide con las referenciadas en tanto en cuanto los dos motivos más referidos por los jóvenes sevillanos son, en primer lugar, la desmotivación, seguido de la falta de tiempo. Sin embargo, nuestro estudio se diferencia de aquellas en el orden de importancia de ambas causas, ya que en los otros estudios, los motivos mencionados invierten su orden.

En relación al género, a diferencia de los otros estudios, los porcentajes de uno y otro sexo son similares, aunque sí coincide con los anteriores en que las chicas son las que suman mayores cifras de abandono.

En función de la edad de las personas, nuestro estudio y los otros estudios revisados registran resultados diametralmente opuestos

En cuanto al nivel académico, nuestros resultados son congruentes con los de las investigaciones referenciadas.
Finalmente, en nuestra investigación hemos aportado un dato no reflejado en las anteriores, éste es, las diferencias entre centro públicos y privados donde hemos comprobado la mayoría del alumnado de los centros públicos suele abandonar la práctica físico-deportiva por desmotivación mientras que la mayor proporción del alumnado de los privados-concertados suelen dejarlo por incompatibilidad con los estudios.

\section{REFERENCIAS BIBLIOGRÁFICAS}

1. Víquez, F., Araya, G. (2007). Determinación de factores relacionados con el abandono de la práctica deportiva en ex-nadadores costarricenses. Rev MHSalud. 4 (2). En www. una.ac.cr/mhsalud. org. Consulta: 30/10/10

2. Serra, J.R., Generelo, E., Zaragoza, J. (2010). Barreras para la realización de actividad física en adolescentes de la provincia de Huesca.Rev.int.med.cienc.act.fís. deporte, 10 (39), 470-483.

3. Pate, R.R., Freedson, P.S., Sallis, J.F., Taylor, W.C., Sirard, J., Trost, S.G. (2002). Compliance with physical activity guidelines: Prevalence in a population of children and young. Annal of Epidemiology, 12 (5), 303-308.

4. Juniper, K.C., Oman, R.F., Hamm, R.M., Kerby, D.S. (2004). The relationship among constructs in the health belief model and the transtheorical model among african-american college women for the physical activity. AJHP, 18 (5), 354-357.

5. Ruiz Juan, F., García Montes, $M^{a} E$ (2002). El abandono y la ausencia de práctica de actividad físicodeportiva de tiempo libre del alumnado de la Universidad de Almería. Un estudio longitudinal. En www.efdeportes.com. Consulta $03 / 11 / 10$

Rev. Ib. CE. Act.Fis. Dep. 
6. Cookburn, C. (2000). Las opiniones de chicas de 13 y 14 años sober la Educación Física en las escuelas públicas británicas. Estudio realizado en los condados de Hampshire y Campbridgeshire. Apunts: Educación Física y Deportes 62, 91-101.

7. Caspersen, C.J., Pereira, M.A., Curran, K.M. (2000) Changes in physical activity patterns in United States, by sex crossectional age. Med \& Sce in Sport and Ex, 32 (5), 1601-1609.

8. Telama, R., Yang, X. (2000). Decline of physical activity from foung to young adulthood in Findland. Med \& Sce Sport Ex, 32 (5), 1617-1622.

9. Van Mechelen, W., Twisk, J.W.R., Post, G.B., Snel, J., Kemper, H.C.G. (2000). Physical activity of young people: Amsterdam Longitudinal Growth and Health Study. Med \& Sce in Sport and Ex, 32 (5), 1610-1616.

10. Steptoe, A., Wardle, J., Cui, W., Bellisle, F., Zotti, A.M., Baranyai, R. (2002). Trends insmoking, diet, physical exercise, and attitudes toward health in european university students fron 13 countries, 1999-2000. Prev Med, 35 (2), 97-104

11. Neumark-Sztainer, D.R., Story, M., Hannan, P.J., Tharp, T., Rex, J. (2003). Factors associated with changes in physical activity: A cohort study of inactive adolescent girl. Archives of Pediatrics \& Adolescent Medicine, 157 (8), 803810.

12. Gavala González, J. (2010). Practical reasons of physical activity of the people of Sevilla. J Sport Health Res. 3(3):169-178.

13. Kimm, S.Y., Glynn, N.W., McMahon, R.P., Voorhees, C.S., Striegel-Moore, R.H., Daniels, S.R. (2006). Self-perceived barriers to activity participation among sedentary adolescent girls. Med \& Sce in Sport and Ex, 38 (3), 534540.

14. Springer, A.E., Kelder S.H., Hoescher, D.M. (2006). Social support, physical activity and sedentary behavior among 6th-

Rev. lb. CL. Act.Fis. Dep. grade girls: A cross-sectional study. Int $J$ Behavioral Nutrition and Physical Act, 3-8.

15. Mota, J., Sallis, J.F. (2002). Actividade física e saúde. Actividade Física e Saúde Factores de Influência Da Actividade Física Nas Crianças e Nos Adolescentes, 71-90.

16. Hernán, M., Ramos, M., Fernández, A. (2004). La salud en los jóvenes. Gaceta Sanitaria: Órgano Oficial de la Sociedad Española de Salud Pública y Administración Sanitaria, 18 (1), 47-55.

17. Chillón, P. Efecto de un programa de intervención de educación física para la salud en adolescentes de $3^{\text {o }}$ de ESO. [Tesis Doctoral]. Granada: Universidad de Granada; 2005.

18. Águila, C., Andújar, C. (2000). Reflexiones acerca del entrenamiento en la infancia y la selección de talentos deportivos. En www.efdeportes.com. Consulta 03/11/10.

19. Salguero, A., Tuero, C., Márquez, S. (2003). Adaptación española del cuestionario de causas de abandono en la práctica deportiva: validación y diferencias de género en jóvenes nadadores. En www.efdeportes.com. Consulta 03/11/10

20. Berry, T., Naylor, P.J., WharfHiggins, J. (2005). Stages of change in adolescents: An examination of self efficacy, decisional balance, and reasons for relapse. J Adolescent Hth, 37 (6), 452-459

21. Del Hoyo, M., Sañudo, F (2007). Composición corporal y actividad física como parámetros de salud en niños de una población rural de Sevilla. RICYDE, (6), 52-62.

22. Montil, M. Determinantes de la conducta de actividad física en población infantil. [Tesis Doctoral]. Madrid: Politécnica de Madrid; (2004).

23. Müller-Riemenschneider, $F$., Reinhold, T., Willich, SN. (2009). Cost-effectiveness of interventions promoting physical activity. $\mathrm{Br} J$ Sport Med 43 (1) 70-76. 